\title{
Host country responses to Black African migrants and refugees living with HIV
}

\author{
Mark Henrickson, Christa Fouché, Cynthia C. Poindexter, Derek B. Brown \\ and Kay Scott
}

The research reported in this article has been conducted by an international research team with extensive expertise in working and publishing in the field of HIV and AIDS. Mark Henrickson is from Massey University; Christa Fouché is from the University of Auckland; Cynthia Poindexter and Derek Brown are from the Graduate School of Social Service at Fordham University in the USA; and Kay Scott is Director of the HOPE Center at St. John's Riverside Hospital in New York, USA. The corresponding author for this article is Christa Fouché, School of Counselling, Human Services and Social Work, University of Auckland, email: m.henrickson@massey.ac.nz.

\section{Abstract}

HIV has never respected national borders. In the context of displaced Africans the pandemic takes on increased significance. This article reports findings from an exploratory study of Black African migrants and refugees living with HIV in New Zealand. While the issues are specific to this country and these participants, they have relevance to all countries hosting newcomers; the need for a shift in meeting a changing demographic will be true for countries experiencing the African diaspora and resulting increase in HIV disease. Thirteen semi-structured interviews highlighted the importance of both African culture and host-country culture on experiences of stigma and decisions to access services. Participants' African heritage played a crucial role in their decision to disclose while the host country culture was reflected in immigration requirements and participants' experiences of care. Findings have relevance for shaping services to meet the needs of the increasing population of migrants and refugees globally.

\section{Introduction}

The African Diaspora continues to necessitate responsive social services in host countries around the world, particularly regarding HIV/AIDS. This paper reports findings from a study of Black African migrants and refugees living with HIV in New Zealand (New Zealand). While the issues emerging from this study are specific to New Zealand, they are relevant to all Western host countries experiencing the African Diaspora. Understanding key concerns and cultural contexts will allow social workers to provide more appropriate and effective services to Black Africans living with HIV, while at the same time encouraging this vulnerable and often isolated group to access relevant and necessary services and support.

As reported by the United Nations High Commission for Refugees (UNHCR), more than 800,000 persons were displaced as refugees across international borders in 2011 (UNHCR, 2011). Social, political and economic disruptions in many parts of Africa have resulted in a 
significant increase in displaced persons, with Africa producing the greatest number of new asylum-seeker applications from any other region (UNHCR, 2011). It can thus be expected that many non-African countries across the globe will host African migrants and refugees and also those living with HIV. HIV has never been a respecter of national borders and in the context of displaced Africans takes on an increased significance. The World Health Organization (WHO) reports that Sub-Saharan Africa accounts for almost 70 percent of the world's population of persons living with HIV/AIDS, so HIV prevalence in African migrant and refugee communities can be expected to reflect that of the countries of origin (WHO, 2012).

\section{The New Zealand context}

New Zealand is a frequent destination for migrants, with $24 \%$ of the resident population born overseas (Statistics New Zealand, 2013). New Zealand also accepts approximately 750 United Nations quota refugees annually (Refugee Services, Aotearoa New Zealand, n.d.). A 2004 Special Zimbabwe Residence Policy, along with a subsequent 2006 waiver allowing HIV-infected Zimbabweans to apply for residency, resulted in nearly 1,000 Zimbabweans applying to remain in New Zealand (Joint United Nations Programme on HIV / Acquired Immune Deficiency Syndrome, 2008). Due to challenges in interpretation of identity and the reliability of country-specific statistics, the exact number of Black Africans in New Zealand is unknown, but estimated to be 12,500 at the end of 2011 (Dickson, Henrickson and Mhlanga, 2012), or about 0.3 percent of the usually resident New Zealand population.

At the end of 2010 a total of 3,474 people in New Zealand had been diagnosed with HIV (AEG, 2011). Since HIV in New Zealand has followed the infection pattern that occurred in most developed nations, the initial response to the disease was launched by gay male communities (Dickson and Davidson, 2006). These successful early initiatives contributed to the relatively low incidence of HIV in New Zealand. A gradual rise in new HIV infections was reported by 1998, and from 2000 to 2005 the number of newly identified HIV infections nearly doubled (Dickson and Davidson, 2006). This increase included more heterosexual transmissions and, significantly, large percentages of this reported heterosexual transmission occurred overseas: 88\% in 2005 and 66\% in 2010 (AEG, 2011). The reported newly infected individuals were predominantly Asian or African migrants (Ministry of Health New Zealand, 2007). These trends have resulted in an increase in the numbers of migrants and refugees living with HIV seeking medical and social services (Worth, Denholm and Bannister, 2003); since 1996 Black Africans have accounted for 19\% of HIV diagnoses, despite their relatively small proportion of the population, and estimated prevalence of HIV in this population is about five percent (Dickson, et al., 2012). Despite relatively small overall numbers of HIV in New Zealand, this trend has signalled important shifts in the nature of New Zealand's HIV health care and social service delivery, dominated by the political and social discourses of white gay men. The need for a shift in meeting a changing demographic will be true for many Western host countries like New Zealand, affected by the African Diaspora and resulting HIV demographic.

This article considers the findings of a study of the lived experiences and social service needs of HIV-infected Black African migrants and refugees in New Zealand. The findings have relevance for social service providers in host nations faced with the growing challenge of meeting the needs of HIV-positive Black African migrants and refugees. The study utilised a general inductive approach (Thomas, 2006) in that it allowed findings to emerge from the 
dominant or significant themes inherent in the raw data. For the purposes of this paper we have clustered themes generally into two major groups, the first related to African culture, and the second related to host-country culture. The first group includes a particular focus on the importance of ubuntu ${ }^{1}$, vertical collectivism and the continuing importance of African culture for our African participants, and also incorporates key themes of stigma about HIV amongst Africans and the decision to disclose an HIV status. In the second group we found the challenges encountered by Black African migrants and refugees living with HIV in their host country. This includes a particular focus on immigration requirements, experiences of care (both formal and less formal), and their experience of stigma.

\section{Methodology}

This qualitative study explored the lived experiences and social service needs of HIV-infected Black African migrants and refugees in New Zealand. The research team comprised researchers from New Zealand and the US. Ethics approval was obtained from the institutional review board of three universities. The interview schedule was developed from literature and refined with input from community partners. Recruitment involved distributing flyers through targeted community-based agencies and hospitals serving people living with HIV and inviting potential participants to contact researchers to arrange an interview. With the assistance and guidance of those community-based agencies, researchers were successful in reaching a highly stigmatised population who would not have been reached otherwise. Fourteen participants agreed to be interviewed, with one withdrawing data from the study shortly after the interview due to confidentiality concerns. Of the 13 remaining interviewees, nine were females and four were males.

As can be expected from a study of this nature, a number of ethical challenges had to be managed, mostly related to confidentiality and risk of harm. We agreed on confidentiality protections with every participant (i.e., whether they were able to receive mail, email or telephone calls from us). Each participant chose their own pseudonym. At each interview we probed for support needed and received, and had social service and medical referrals at hand. All but one of the interviews, including the three telephone interviews, were recorded and transcribed; one interviewee did not want his voice recorded, but allowed the researcher to take verbatim notes. Telephone participants were interviewed by appointment, so participants had the opportunity to ensure the privacy of their environments. Participants also had the right to edit the transcripts of their interviews; this allowed them to edit the transcripts if necessary to protect their confidentiality and their families' safety. This partially mitigated the dynamics of interviews conducted by phone rather than in person.

Data were thematically analysed with the aid of ATLAS.ti@. Three levels of coding were used: descriptive, interpretive and pattern coding (Miles and Huberman, 1994), leading to the identification of interconnected themes. The transcripts from the interviews were coded separately to ensure accuracy of coding. Recurring codes were clustered to develop themes. The delineation of African and host-country culture arose from the clustering of codes into themes.

Ubuntu is defined both as the moral quality of a person and a philosophy according to which persons are connected (Gade, 2012). 


\section{Findings}

The discussion will firstly focus on the findings pertaining to the African culture, with particular emphasis on the opposing and conflicting experiences of ubuntu and stigma. The discussion will then turn to the experiences Black African migrants and refugees living with HIV face in their host country, including the value of care and the challenge of stigma.

\section{Home country culture}

\section{Ubuntu}

African identity is intricately linked to community; what is best for the community is best for the individual. This is probably best captured in the African concept of ubuntu - defined both as the moral quality of a person and a philosophy according to which persons are connected (Gade, 2012). Caldwell-Harris and Aycicegi (2006, p. 335) explain that New Zealand is a country with 'horizontal individualism', where the 'individual is allowed to pursue their own goals without undue obligation to their in-group'. They note the shortcoming of this is social isolation. Most African cultures would be considered vertical collectivist, where individuals subordinate their own desires to those of the group leaders. Study participants noted stark differences between the collectivist nature of African culture and individualistic Western cultures, as well as frustration with having to 'live a lie' in both cultures. The following statements illustrate this, where first Rute contrasts her home culture with her refugee culture, and where Mary explains the shared nature of her home culture:

In our culture we grow together, we are close; we can live together forever. If you are my age and not married, it doesn't matter. We live together. We are happy to be together. It is very hard for us to live here, like you guys (Rute).

When you want people to come here, play, sleep over, that's African. They can come, every time sleep over. But [now] you have to stop everything. I am not a secret woman. No. I want to laugh, say come and sleep over ... but it's like something stopped inside me. We are not like everybody (Mary).

Almost all of the participants in this study reported experiencing HIV stigma within their own cultural communities, and mostly chose to remain separate, silent and hidden. This self-imposed isolation has had an inevitable personal, social and health impact on their lives, as Adele and Joe reflect in the excerpts below.

My own community, they know but they want to pretend like they don't know. Because back home I believe every African has got a relative, a family friend or a friend who has been affected by HIV but here they just don't want to identify themselves to support each other. [This is] because of the stigma and oh well, if you have HIV it means you have been promiscuous or you've been doing things that are not good and maybe it's pay-back for your sins or whatever - that you were doing. It's kind of very lonely (Adele).

Unless maybe you [Africans to Africans] are discussing an issue like something you've read about in the paper or something like that but just talking about it [HIV] is very rare. I don't recall a time when I was just talking about HIV with someone (Joe).

African culture, a source of strength and support, is not merely a source of isolation and gossip. Nine participants described positive statements of African culture because of its 
emphasis on the importance of groups, such as families (Adele, Anna, Joe, Lisa, Mary, Pat, and Rute) and communities (Adele, David, Jane, Mary, and Rute). Mary highlighted the irony of this; she misses being in a social culture, but realises that being social also means talking about group members:

I miss Africa because in Africa, you're social with everyone, but in New Zealand everyone is busy... if it was in Africa, everyone would start to gossip; start talking, she's HIV, or laugh on you. But here, nobody knows your life (Mary).

The role of such social capital is widely debated within the context of public health and HIV in particular (Szreter and Woolcock, 2004). In some contexts high levels of social capital and community cohesion might facilitate more effective collective responses to the epidemic, and a lack of social capital may serve to exacerbate HIV infection and limit the effectiveness of control efforts. In other contexts, group membership can be a risk factor for HIV infection and contribute to stigma (Pronyk, et al., 2008). In this context we found that the social capital of African communities was two-edged regarding migrants living with HIV: it provided an expectable community of support, but because of the social expectations and norms of the community, was also, through gossip and stigma, a source of exclusion.

\section{Stigma}

Stigmatisation of the social groups with which they are associated remains a troubling reality in the context of the African Diaspora (Grierson, et al., 2008). Communities, families and individuals within these populations effectively live with the dual stigmas of not only living with HIV, but also being perceived as an HIV-affected migrant community. Findings from the New Zealand study by Henrickson, Dickson, Mhlanga and Ludlam (2013a) highlighted that many participants felt that they were stigmatised as being HIV positive just because they are Black Africans. Since the emergence of the HIV pandemic, stigma and discrimination have frustrated efforts to halt the spread of the disease. Despite the acknowledgment of and commitment to address the issue by governments and international organisations such as the WHO, UNAIDS, UNHCR and others, stigma remains a defining feature of living with HIV. It was very evident from the current project's data that HIV-related stigma had a far-reaching effect on participants' lives. A very powerful statement from one of our participants is illustrative of such an experience: 'You live a lie life' (Mary).

Knowing that stigma is often the consequence of HIV infection, people with high concerns for stigma may be less likely to disclose their HIV status to others. Twelve of the 13 participants spoke of keeping secret their own and/or their children's HIV status. The following comment from Anna powerfully reflects this:

... it's hard when [my son's African] friends come and they sleep over. I just hide the medication. It's living a lie really (Anna).

Ivy reports similar secretive behaviour with her sister:

I was talking to my sister - she's back home. She is saying she was sick and I was saying 'go and have an HIV test, maybe you are not okay'. They say 'oh I can't'. But I can't tell them. I can't tell that I know the procedure, don't worry, you'll be fine. I can't do that. I can't comfort her. I can't really tell her (Ivy). 
Evidence is strong that internalised HIV stigma may play a crucial role in the emotional distress experienced by many people living with HIV across cultures (Kalichman, et al., 2009). Often the choice these individuals face is one of disclosure versus social support; they cannot have both. Some refrain from disclosing their diagnosis, even though nondisclosure causes social isolation. Others hope that making their status known will benefit others. Migrant populations affected by HIV are particularly at risk for stigma (Dickson and Davidson, 2006; Henrickson, et al., 2013a).

\section{Host country culture}

\section{Immigration}

The arrival of African migrants in New Zealand beginning in 1992 coincided with and was caused by significant political, economic and social upheaval in many parts of the African continent (Worth, et al., 2003). Almost all migrants experience multiple stresses that can affect their mental wellbeing; rates of mental illness are increased in some migrant groups who experience the loss of cultural norms, religious customs and social support systems (Fazel, Wheeler and Danesh, 2005). Resettlement support as a planned process of change seeks to assist refugees with the challenges of early integration and to empower them to become independent, fully contributing members of their new communities. Integration requires amongst other things, opportunities for training and skills development; the availability of services tailored to vulnerable groups; and the coordination and engagement of all relevant government authorities (UNHCR, 2013, p. 12). The responses related to resettlement by respondents in this research included their ability to access support and in particular mainstream social services tailored to people with HIV. Pat was one of the few participants who indicated a reluctance to make use of one of the support services - although she had good medical support:

I'm really ashamed. I don't know what it would be like if I go there, what I will say...I don't feel comfortable, so I never have been there. I have always been like a private person so it's really hard for me to go into a crowd of people and start talking about my life (Pat).

Conversely, several participants talked about how living with HIV disease encouraged them to access social support from others who are also HIV positive. These relationships served as opportunities for mutual support. It is in these new communities that HIV became normalised and destigmatised. The notion of meaning-making by non-migrant Africans with HIV has been extensively debated in the literature (Nattabi, Li, Thompson, Orach and Earnest, 2011; Kyakuwa and Hardon, 2012). Henrickson, et al. (2013b), highlight that within the African Diaspora, however, migration adds a stressor that necessitates that Black African migrants and refugees use their resilience to extend their strategies for meaning-making and garnering support. The process for extending strategies of meaning-making has not been identified in Africans with HIV who continue to live in Africa. One participant commented:

I have joined [Agency Name]. They are very supportive and that's where I've managed to meet some friends who are also HIV positive as well... when I am with them, I can be myself. I can talk about anything... I am free because they've been there and they know what I'll be talking about (Adele).

The immigration process was characterised as difficult by many participants who had travelled to New Zealand without members of their nuclear and extended families to escape 
conflict or an ailing economy. In addition to the stress regarding uncertainty of future residency, participants also acknowledged support received from others in the process. Anna reflected on these experiences as follows:

Most of the time I rely on people like [support worker from HIV service], and also [names of agencies providing peer support]; I used to contact them a lot with some issues (Anna).

Adele similarly commented on the support from people in the same position:

... Those people... they are also positive, they know what they've been through. We are going through the same thing so we can relate and comfort each other though we are in different phases. Some have got support and they've managed to overcome other things so they can actually give you good support as well (Adele).

Again, participants demonstrated a challenging stance amidst these difficult circumstances, as in this comment by Adele:

I wish the Government can also look at how I am contributing to their economy and community....I want to work because I am still able to work until when I can't work. But it's so hard, so hard. It also reminds me that I am different here. I can say I am just like anyone else but because I have got HIV I am different. I can't live where I want to live. I can't do the things that I want to do. I am discriminated against (Adele).

\section{Experiences of care}

Over the past decade, advances in antiretroviral therapies (ART) have greatly assisted people infected with HIV to live longer and healthier lives. Several participants reflected on the high quality of related care in New Zealand (as opposed to their African country of origin) and were very positive about the health care available to them. As highlighted in the discussion on discrimination above, some participants reported negative experiences in the health sector in general, but this did not apply to HIV-related care. The majority of the participants report receiving their HIV-related medical care through a system that included a general practitioner working with an HIV-specialist, and that this worked very well. One participant's comment was particularly heartfelt:

They care about me. I didn't see it from other people in all of my life; I didn't see this kind of thing. They are very special ...they are very kind. I say God thank you (Rute).

Another highlighted the efficiency of the support:

It was very fast, my doctor made an appointment (and) the same week I went to a specialist (David).

Participants identified the HIV specialists and their staff as one of the major sources for accurate information on the disease. Ivy stated that specialists supply them with 'facts'. Jane and Paul noted that specialists update them on drugs or other new information about the virus, while nurses are also valued for their ability to educate about the disease. Specialists and staff are also highly valued for their support and for the fact that they do not stigmatise those with HIV and maintained confidentiality about their HIV status, as indicated in these statements by Paul and Ivy: 
They will simply explain everything in full details and then you understand. It's only my medical specialist and his supporting staff [that I talk to] ... (Paul).

The doctors are no problem. So far I haven't had any cause to be scared information will come out (Ivy).

Social workers appear to successfully fulfil roles as a source of referrals to medical and social services, and providing a capacity to listen:

I had a good relationship with my social worker. She helped me a lot. I started opening up and there were a lot of things I didn't know, that I could have access to (Anna).

But all of this did not prevent the apparently inevitable experience of stigma.

\section{Stigma}

Some of these participants frequently reported incidents in New Zealand of employment and medical discrimination, and spoke of being socially isolated, and having their confidentiality violated. Some of the comments by Adele, David and Anna are indicative of these experiences:

When I discovered I was HIV positive, the one that did the test, she told me that I've got to go and tell my employer. My employer needs to know, because I may infect the people that I work with (Adele).

...even getting jobs, the ones [screening questions] on the medical - when you say you are HIV they don't want to take you. I know it could be illegal but you see, here is someone who is empowered to employ. He will only say no, sorry. Already [the discrimination] it's there (David).

Me and my son were put in a single room and we were put in isolation and they had all these stickers outside the door like 'this patient is in isolation' ... What I understand now was it was hospital protocol [for tuberculosis, but interpreted as related to HIV] but nothing was explained to us so I had no idea what was going on. I had no-one to talk to and I was really scared of asking questions (Anna).

As will be discussed later, participants experienced a high quality of HIV-related care in New Zealand. However, participants reported discrimination from other medical professionals. Adele and Julius made the following comments, illustrative of this:

The dentist I see here, on their questionnaire they asked me if I have a communicable disease, so I opened up and wrote that I am HIV positive and when they read it, they just did see me quickly and hurried me off and within two weeks the filling they did just came off meaning they didn't do a thorough job because they thought that they would get it from me (Adele).

Stigma is the same across the board. I remember I had to get a tooth extraction, and there's question on the medical form about HIV. I answered the question and submitted the form to the receptionist. ... I saw her response. She reacted - I saw her facial expression. I saw her whispering to her colleagues around the office (Julius).

It is interesting that even when the problems the participants experienced were not directly related to HIV (e.g. tuberculosis, a failed dental filling), they were interpreted as related to HIV. Attitudes affect prevention, intervention and quality of life in people living with HIV disease (Grierson, et al., 2008). Our participants also made comments that were evident of 
resilience and courage and took a stance that challenges the stigma that they experience. Mary raised both a cost-effective and social justice argument:

I wrote a letter to them. I said if you calculate the amount of money that I pay for tax and my cost in hospital, I think it balances out in the long run. They will still end up benefitting from the tax that I am paying (Mary).

As highlighted by Henrickson, et al., (2013b), HIV stigma holds African migrants hostage. They are holding back from forming alliances with other African refugees - short-circuiting their chances for support and community - out of realistic, necessary self-protection and self-preservation.

One of the most important ways that stigma shaped participant responses was around the decision to disclose their HIV status. Accounts of navigating the terrain of personal relationships and decisions about if, when, how and to whom they will disclose, were disheartening, but unfortunately not isolated, as highlighted in the literature. People living with HIV remain mired in conflictual relationships between persons living with HIV and the untested (Campbell, et al., 2011). Pat and Mary's comments illustrate this tension:

... revealing my status..; that's one thing that really upsets me when I start thinking about it.

Like how are people going to take me, how are they going to react? (Pat).

I don't choose to be alone but I have to be that to keep my privacy (Mary)

Some wanted to tell their stories in order to make things better, not only for themselves but also for others in a similar situation. Lisa's comment is one example:

Probably in future I'll come out, for me it's like nothing for me to be quiet about. ...it's a huge negative impact but it can be turned into a positive input into other people's lives; by sharing the experience (Lisa).

\section{Discussion}

The recent arrival of Black African migrants and refugees to New Zealand has altered this country's HIV demographic considerably. The experiences reported by migrants and refugees living with HIV in this study suggest that a layered response from social service providers is required in New Zealand, and any country hosting migrants and refugees living with HIV. This is supported by findings from a number of studies in the US and UK on migrants living with HIV (see e.g. Foley, 2005; Gardezi, et al., 2008) and holds significant implications for social workers in other national environments.

Our findings highlight the need for greater sensitivity and knowledge on the part of social service, health, dental and other allied health care providers in order to protect the legal and human rights of participants who reported frequent and gross breaches of their privacy and confidentiality. At the moment, the response in New Zealand, available primarily through HIV-dedicated service organisations and organisations working with sexual and gender minorities is aimed at HIV education and health promotion in refugee communities and service provider training and support. HIV-related education should be provided to health professionals and allied health and ancillary care workers who work with African 
communities outside the offices of HIV specialty providers. General health and social service practitioners with adequate knowledge about HIV and HIV-related service provision in rural and remote areas is a concern. Efforts are needed to standardise care outside of the main regions and across health and social services. We suggest this may be best achieved by re-focusing professional health education so that this vulnerable population can expect appropriate, effective and targeted interventions.

We also urge that social workers increase their understanding of the strengths and challenges of the African culture. While it is far beyond the scope of the present paper to present and discuss the complexities of the many African cultures, improving social worker knowledge can be done by enhancing existing social work education curricular offerings, and also by enhancing continuing professional development opportunities both nationally and within agencies. The need for more culturally specific support services is emphasised by both Foley (2005) in a study conducted on the cultural and structural barriers African women face in the area of HIV prevention, testing and treatment, and by Gardezi, et al. (2008) in a study on factors that influence African and Caribbean communities' responses to HIV prevention practices and access to treatment and support services. Both these studies conclude that culturally appropriate education about HIV prevention and treatment needs to be developed for African new settlers, and healthcare providers need to understand the experiences, fears and concerns of this population. These findings are consistent with those reported in Gardezi, et al. (2008) that HIV-positive participants spoke highly of the healthcare they received but that discriminatory attitudes from some healthcare and service providers create a reluctance to access health and social services.

\section{Conclusion}

It is evident from our data that peer support organisations were highly valued and often provided the only sense of community to participants. Even though the decision to access these organisations was difficult and fraught with anxiety about disclosure and privacy, the focus for social workers should in the short term include promoting the safety, availability and accessibility of peer support organisations. Establishing, supporting and gently but persistently encouraging people to safe places where Black African migrants and refugees living with HIV can come together and create new purpose in their lives is an essential aspect of holistic HIV care. This is particularly important for collective cultures and the spirit of ubuntu. A concerted effort is needed, in which social workers can play a significant role, to develop peer support organisations accessible to specific cultural needs in ensuring effective services to this population.

Raising awareness about HIV and associated stigma is one of the most important and immediate responses. HIV service organisations and related agencies must address and reduce the stigma and discrimination experienced by Black African migrants and refugees living with HIV, particularly during a period of resettlement. Some migrant and refugees groups are easily identifiable, and only legislative and political advocacy will prevent entire communities from being associated with HIV by residents of the host country. Respondents in one study (Gardezi, et al., 2008, p. 723) reported on the 'pathologizing of Black bodies as repositories of HIV and other diseases'. Expanding the capacity of HIV service organisations to work with new migrant communities has become more urgent than ever. An HIV infrastructure that has grown from and for gay male communities can 
be helped to see the commonalities in the experiences of social exclusion, marginalisation and stigma, and to transfer lessons about managing responses to those experiences to new migrant communities. This includes the use of participatory action methods to support Black African migrants to have a voice in the planning of HIV services in New Zealand, as (white) gay men have done in the past. Moreover, global attention to the social and structural sources of HIV vulnerability is essential. Although organisations such as the World Health Organization, United Nations and others provide important resources to address these issues, the social work profession in New Zealand must maintain an active voice for strategic attention to the determinants of HIV. The complex links between migration and HIV are not well understood (Sosklone and Shtarkshall, 2002), but it is acknowledged that there has been a shift from individual HIV risk to social vulnerability - particularly pertinent in migrant populations, and these need to be emphasised. Greater community awareness and expanded efforts to tackle housing, poverty, racism and settlement issues are called for by Gardezi, et al. (2008).

The African Diaspora necessitates the need for responsive social services in host countries. While HIV is but one challenge faced by Black African communities in New Zealand, it is a challenge that brings into sharp relief the multifarious challenges and strengths that these communities bring with them. In order to contribute to a collective holistic response, social workers, researchers and social and health service providers in New Zealand and other host countries must continue to evolve evidence-based services in response to the increasing numbers and needs of the Black African communities.

\section{References}

AEG. (2011). AIDS-New Zealand. Issue 67, Dunedin: New Zealand: University of Otago Medical School, AIDS Epidemiology Group.

Caldwell-Harris, C. L., \& Aycicegi, A. (2006). When personality and culture clash: The psychological distress of allocentrics in an individualistic culture and idiocentrics in a collective culture. Transcultural Psychology, 48(3), 331-361.

Campbell, C., Skovdal, M., Madanhire, C., Mugurungi, O., Gregson, S., \& Nyamukapa, C. (2011). 'We, the AIDS people. . .': How antiretroviral therapy enables Zimbabweans living with HIV/AIDS to cope with stigma. American Journal of Public Health, 101(6), 1004-1010.

Dickson, N., \& Davidson, O. (2006). HIV prevention in New Zealand - still room for improvement. New Zealand Medical Journal, 119(1243). Retrieved from: http: / / www.New Zealandma.org.New Zealand/journal/119-1243/2250/.

Dickson, N., Henrickson, M., \& Mhlanga, F. (2012). Africa New Zealand count: An estimate of currently resident and HIV positive Africans in New Zealand. Report completed for the Health Research Council of New Zealand (Contract 11/965) and the Ministry of Health. Auckland, New Zealand: Health Research Council of New Zealand.

Fazel, M., Wheeler, J., \& Danesh, J. (2005). Prevalence of serious mental disorder in 7000 refugees resettled in western countries: A systematic review. Lancet, 365(1), 309-314.

Foley, E.E. (2005). HIV / AIDS and African immigrant women in Philadelphia: Structural and cultural barriers to care. AIDS Care, 17(8), 1030-1043.

Gade, C.B.N. (2012). What is ubuntu? Different interpretations among South Africans of African descent. South African Journal of Philosophy, 31(3), 484-503.

Gardezi, F., Calzavara, L., Husbands, W., Tharao, W., Lawson, E., Myers, T., \& Adebajo, S. (2008). Experiences of and responses to HIV among African and Caribbean communities in Toronto, Canada. AIDS Care, 20(6), 718-725.

Grierson, J., Thorpe, R., Pitts, M., Hughes, T., Saxton, P., Smith, J., Smythe, E., \& Thomas, M. (2008). HIV futures New Zealand2/Mate araikore a muri ake nei (Tuarua). Melbourne, Australia: The Australian Research Centre in Sex, Health and Society, La Trobe University.

Henrickson, M., Dickson, N., Mhlanga, F., \& Ludlam, A. (2013a) Africa New Zealand Care: A report on knowledge, attitudes, behaviours and beliefs about HIV among Black Africans living in New Zealand. Report completed for the Health Research Council of New Zealand and the Ministry of Health (Contract 11/965). Auckland, New Zealand: Health Research Council of New Zealand.

Henrickson, M., Brown, D.B., Fouché, C., Poindexter, C.C., \& Scott, K. (2013b). 'Just talking about it opens your heart': Meaning-making by black African migrants and refugees living with HIV. Culture, Health $\mathcal{E}$ Sexuality: An International Journal for Research, Intervention and Care, DOI:10.1080/13691058.2013.790076. 
Joint United Nations Programme on HIV/ Acquired Immune Deficiency Syndrome. (2008). UNGASS Country Progress Report: New Zealand. Geneva, Switzerland: UNAIDS.

Kalichman, S., Simbayi, L., Cloete, A., Mthembu, P., Mkhonta, R., \& Ginindza, T. (2009) Measuring AIDS stigmas in people living with HIV / AIDS: The internalized AIDS-related stigma scale. AIDS Care, 21(1), 87-93.

Kyakuwa, M., \& Hardon, A. (2012). Concealment tactics among HIV-positive nurses in Uganda. Culture, Health and Sexuality, 14(Suppl.1), S123-S133.

Miles, M.B., \& Huberman, A.M. (1994). An expanded sourcebook: Qualitative data analysis. Thousand Oaks, CA: Sage.

Ministry of Health New Zealand. (2007). AIDS - New Zealand (Issue 59). Wellington, New Zealand: Author.

Nattabi, B., Li, J., Thompson, S.C., Orach, C.G., \& Earnest, J. (2011). Factors associated with perceived stigma among people living with HIV / AIDS in post-conflict northern Uganda. AIDS Education and Prevention, 23(3), 193-205.

Pronyk, P.M., Harpham, T., Morison, L.A., Hargreaves, J.R., Kim, J.C., Phetla, G., Watts, C.H., \& Porter, J.D. (2008). Is social capital associated with HIV risk in rural South Africa? Social Science \& Medicine, 66(9), 1999-2010.

Refugee Services Aotearoa New Zealand. (not dated). Refugees and New Zealand: Quota programme. Retrieved from: http:/ / www.refugeeservices.org.New Zealand/refugees_and_new_zealand/quota_programme.

Sosklone, V., \& Shtarkshall, R.A. (2002). Migration and HIV prevention programmes: Linking structural factors, culture and individual behaviour - an Israeli experience. Social Science and Medicine, 55(2002), 1297-1307.

Statistics New Zealand. (2006). Quick stats. Retrieved from: http:/ / www.stats.govt.New Zealand/Census / 2006CensusHomePage/QuickStats.aspx.

Statistics New Zealand. (2013). New Zealand as a village of 100 people. Retrieved from: http: / / www.stats.govt.New Zealand / Census / 2013-census / profile-and-summary-reports / 2013-census-infographic-New Zealandvillage.aspx.

Szreter, S., \& Woolcock, M. (2004). Health by association? Social capital, social theory and the political economy of public health. International Journal of Epidemiology, 33, 650-667.

Thomas, D. R. (2006). A general inductive approach for analyzing qualitative evaluation data. American Journal of Evaluation, 27(2), 237-246.

United Nations High Commissioner for Refugees. (UNHCR). (2011). A year of crisis: UNHCR global trends 2011. Retrieved from: http:/ / www.unhcr.org/4fd6f87f9.html.

United Nations High Commissioner for Refugees (UNHCR). (2013). UNCHR projected global resettlement needs 2013. Retrieved from: http:/ / www.unhcr.org/5006aff49.html.

World Health Organization. (2012). HIV/AIDS. Retrieved from: http:/ /www.who.int/topics/hiv_aids/en/.

Worth, H., Denholm, N., \& Bannister, J. (2003). HIV/AIDS and the African refugee education program in New Zealand. AIDS Education and Prevention, 15(4), 346-356. 